\title{
Give Me a Break
}

Design for Communication Among Family Caregivers and Respite Caregivers

\section{Flannery Currin}

Earlham College

Richmond, IN, USA

fhcurrin15@earlham.edu

\section{Aehong Min}

Indiana University Bloomington

Bloomington, IN, USA

aemin@iu.edu

\author{
Gustavo Razo \\ University of California, Merced \\ Merced, CA, USA \\ grazo@ucmerced.edu
}

\section{ABSTRACT}

This study focuses on solutions to issues that arise from gaps in communication between primary family caregivers of older adults and respite caregivers. We collected data through 18 semi-structured interviews with primary family and respite caregivers and qualitatively analyzed the interviews to extract common needs. Participants identified three main needs that our designs address: building trust through status updates, learning routines \& care management, and accessing technology. Based on those needs, we designed a prototype of an application which connects primary family caregivers with respite caregivers and facilitates communication between the involved parties. This design can serve as a framework for future work designed to improve elder care in general, the well-being of caregivers, and the effectiveness of respite care.

Permission to make digital or hard copies of part or all of this work for personal or classroom use is granted without fee provided that copies are not made or distributed for profit or commercial advantage and that copies bear this notice and the full citation on the first page. Copyrights for third-party components of this work must be honored. For all other uses, contact the owner/author(s)

CHI'19 Extended Abstracts, May 4-9, 2019, Glasgow, Scotland UK

(C) 2019 Copyright held by the owner/author(s).

ACM ISBN 978-1-4503-5971-9/19/05.

https://doi.org/10.1145/3290607.3309687 


\section{Key Definitions}

Primary family caregiver: an individual who is not necessarily a trained care professional who provides care for a family member or other loved one. Here, we define primary family caregiver as the person who provides or coordinates the majority of the care for a loved one.

Respite care: provides primary family caregivers with a temporary break from caregiving duties. In this study, most respite caregivers were care professionals or training to become care professionals and most often provided care in their clients' homes. However, some were family members who helped the primary family caregiver occasionally, and some worked with clients in other types of care facilities.

Degenerative conditions: medical conditions such as Alzheimer's or Parkinson's that become more severe over time and require increasing amounts of attention from caregivers as the condition progresses.

\section{KEYWORDS}

Caregiver; primary family caregiver; respite care; older adults; trust management; information sharing

\section{ACM Reference Format:}

Flannery Currin, Gustavo Razo, and Aehong Min. 2019. Give Me a Break: Design for Communication Among Family Caregivers and Respite Caregivers. In CHI Conference on Human Factors in Computing Systems Extended Abstracts (CHI'19 Extended Abstracts), May 4-9, 2019, Glasgow, Scotland UK. ACM, New York, NY, USA, 6 pages. https://doi.org/10.1145/3290607.3309687

\section{INTRODUCTION}

In 2014, 34.2 million people in the U.S. offered unpaid care to older adults. Caregiving is a longterm obligation for most of those family caregivers, and it is associated with caregivers' physical, physiological, and/or mental problems [9]. To reduce the burdens of caregiving and maintain the level of care for older adults, it is essential for primary family caregivers to be able to reach out for help as they need it. Using respite care services allows primary family caregivers to take a break from caregiving and take care of their own social, emotional, and physical needs [5].

Respite care may allow primary family caregivers to relax or have a break from their caregiving work regularly or in emergency situations [6]. However, some caregivers are reluctant to reach out for this care because of embarrassment about needing help, uncertainty about where to look for help, mistrust of the quality of care their loved one would receive, and financial limitations [8].

Information Communication Technology (ICT) has the potential to not only provide a way to reduce the barriers to respite care, but also to offer effective and appropriate methods for primary family and respite caregivers to cooperate, coordinate, and communicate to allow primary family caregivers to have a proper break, which improves both their and their care recipient's health $[1,12]$. Our research focuses on understanding the issues caregivers face and how information sharing through ICTs could temper issues of trust and improve the quality of short-term care work. In this paper, aiming to provide a user-centered design for those caregivers, we introduce our findings from interviews with 18 caregivers and present our prototype of an application that addresses the needs participants discussed.

\section{RELATED WORK}

Trust is a major component of effective communication and has been studied and addressed from a variety of academic perspectives including theology, philosophy, and sociology [4]. Prior research addressed issues of trust between patients and healthcare professionals [10]. However, little research has focused on technology that supports respite care specifically and fosters trust between primary family and respite caregivers. Sharing information through certain media (e.g., "visit notes") can impact primary family caregivers' trust in care professionals [2]. Technology facilitating communication 
"I feel that nobody can take care of her as much as I will do. And I cannot predict what a stranger can do. So I prefer to be available at all times to take care of her needs."

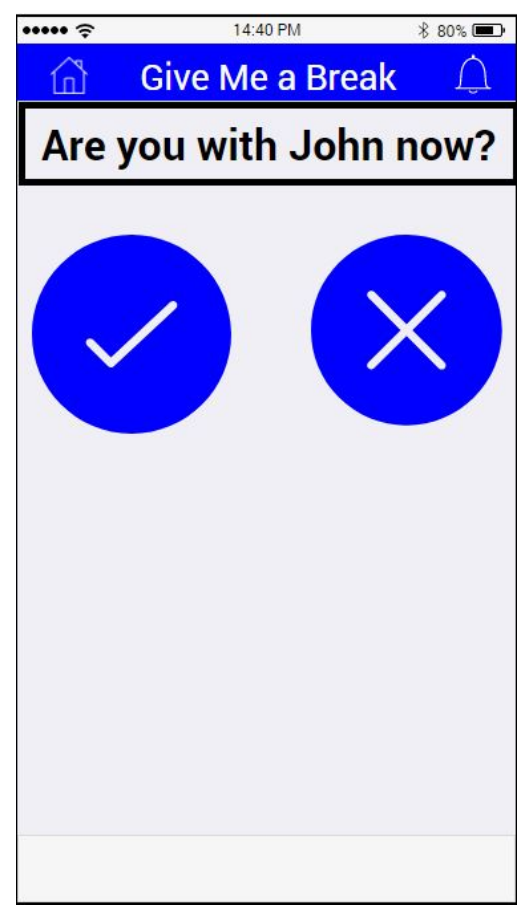

Figure 1: The initial check-in page for respite caregivers allows them to alert primary family caregivers when they arrive for a visit. between primary family caregivers and medical professionals has helped address some issues with tracking a patient's medical history and providing medical professionals with appropriate updates. Foong et al. focused on facilitating the transfer of knowledge between volunteer caregivers in hopes of cutting down on the burden of training programs for volunteer positions with high turnover rates [3]. A similar approach could facilitate information transfer between respite caregivers in situations where a primary family caregiver needs to coordinate care with multiple respite caregivers. Our investigation extends this work to explicitly include respite caregivers in ICT design considerations.

\section{METHODS}

After obtaining IRB approval, we recruited participants through local retirement communities, support groups, respite care agencies, and other local institutions. We recruited a total of 18 participants (17 female, 1 male) who ranged in age from 19 to 80 . They consisted of 7 primary family caregivers, 8 respite caregivers, and 3 participants who had experience as both. We conducted hour-long semi-structured interviews. During the interviews, participants were encouraged to discuss issues or concerns they had during their respite care experiences, but especially those related to information sharing.

Using open coding and affinity diagramming [7,11], we coded the interview transcripts to uncover common and insightful issues. From the primary family caregivers, we looked specifically at what caused caregivers to decide whether to use respite care services, and what issues those of them who had tried respite care services ran into. From the respite caregivers, we focused on what kinds of information they needed to collect from families and the challenges they experienced in obtaining that information which long-term caregivers might not face.

We then iterated on designs, referencing the analyzed data. Focusing on how to address three main concerns, we brainstormed design ideas, narrowed down the range of designs by establishing personas and scenarios, and wireframed and sketched screen designs. We finally designed a prototype that could meet key needs of both primary family and respite caregivers, allowing them to share information with one another easily and regularly.

\section{NEEDS \& DESIGN SOLUTIONS}

We identified three major needs participants discussed which our design directly addresses: (1) building trust through status sharing, (2) learning routines \& care management, and (3) accessing technology. We explicitly incorporated each of these needs into our designs.

\section{Building Trust Through Status Sharing}

Primary family caregivers claimed caring for a loved one with a degenerative condition can make trust more difficult because the care recipient's condition can impact their ability to accurately communicate what problems emerged during a visit. One participant who worked as a care professional herself 
"Ask [primary family members] as many questions as you can, say, 'I see this person, your daughter, your son, your mother, your father doing this action or getting upset when we try to talk to them about this or do this. Can you fill in those gaps? Can you help explain why they do that?' And sometimes they would have answers, sometimes they wouldn't."

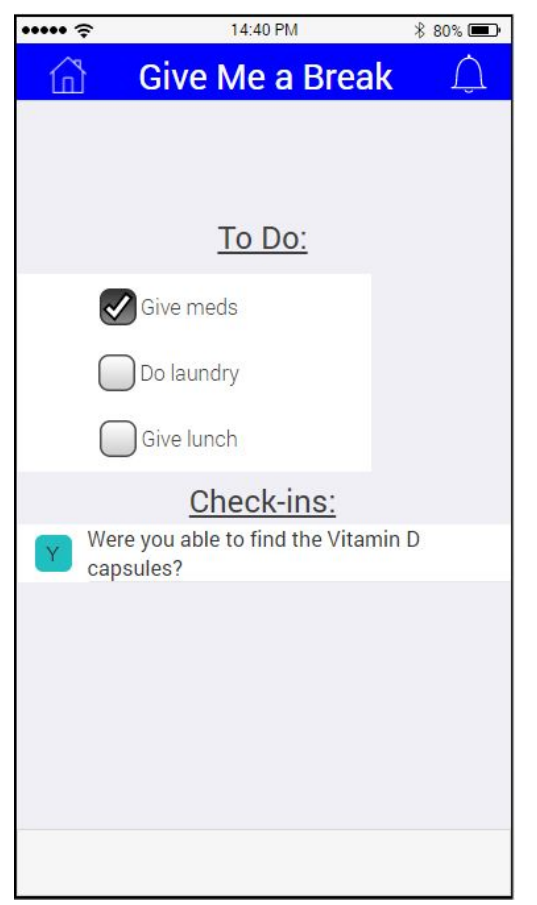

Figure 2: Respite caregivers see this screen after checking in. They can answer questions and check off to-do list items which the primary family caregiver can check on their app if they want an update. went as far as to say that if she had hired a stranger to help care for her mother with Alzheimer's instead of a friend, she would have monitored her house to see exactly what was going on. Hearing a professional express that sentiment shows that respite caregivers are aware of trust issues and see merit in them. However, many of the respite caregivers we interviewed shared experiences in which their job was especially difficult because of trust issues based on pre-conceived notions of paid caregivers, and they also expressed concerns about being monitored by sensors or cameras.

Easy Check-In. To balance primary family caregivers' need to know what is happening with their loved one and respite caregivers' need for privacy, our design provides easy check-in options. If primary family caregivers cannot be at home when a respite caregiver arrives, the respite caregiver can navigate to the check-in page and press one button to notify the primary family caregiver (See Figure 1). This step is necessary before the respite caregiver can access their to-do list in order to encourage regular update habits. However, it does not use GPS-tracking capabilities because respite caregivers reported concerns about privacy and issues checking in for work when they cannot find a strong enough internet signal at a client's home. This simple design also keeps primary family caregivers in the loop without overwhelming them with too much information.

To-Do Checklist. As an extended function of the check-in screen, the respite caregiver can see specific yes-or-no questions from the primary family caregiver as well as a checklist of tasks the primary family caregiver expects them to complete during their visit, as shown in Figure 2. The primary family caregiver can check the same page and see what progress has been made on the checklist or ask a new question without having to send a direct message. The primary family caregiver has control over what content this section displays, but it is organized in such a way that respite caregivers do not have to step away from their caregiving duties to answer calls or messages to provide updates.

\section{Learning Routines \& Care Management}

Some primary family caregivers claimed that respite caregivers sometimes struggled to learn care recipients' routines and conditions. Respite caregivers also mentioned that they need to make an effort to understand their clients, especially during the first few visits. As respite caregivers are usually temporary or short-term caregivers, they do not have much time to become familiar with care recipients' medications, conditions, and routines or learn how a significant change in a care recipient's life might impact them. Moreover, respite caregivers who provided care to multiple care recipients at a time often claimed their clients rotated every few weeks, which could make learning care recipients' specific routines an even slower process.

Case File. The case file screen allows respite caregivers to find important information about care recipients related to medications, routines, and previous visits. Primary family caregivers can leave 
"What we had was essentially a notebook. It was kept in our kitchen, and I would write on here, 'This is when she had her last medication,' or 'This is when she had eaten last.' Or maybe she complained that her stomach's hurting, and might write, 'It seems like she's getting a cold. If she runs a fever, call me." "

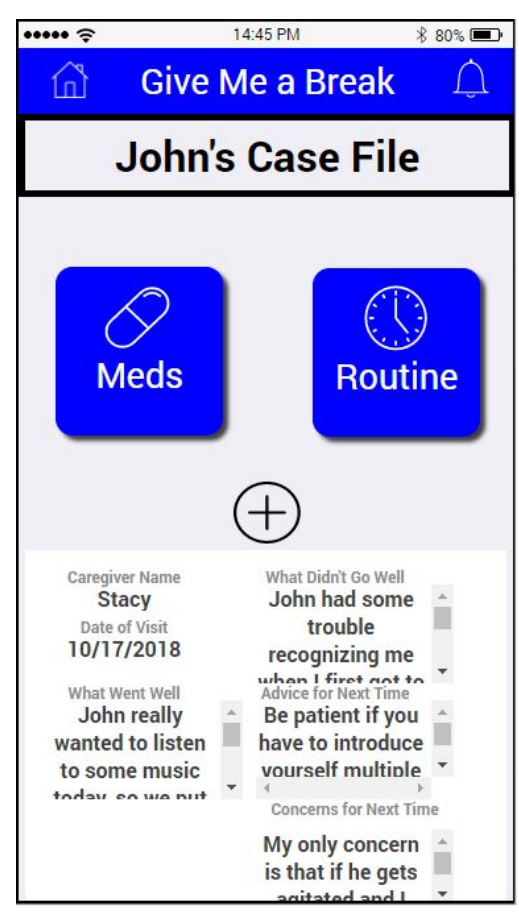

Figure 3: A care recipient's "case file." This screen allows respite caregivers to check a client's medications, routine, and log entries from previous visits. They can create a new log entry by pressing the plus button. information about medications (where they are, how many times they should be taken, etc.) and basic routine (activities, meals, etc.). After a visit, respite caregivers are encouraged to answer four questions about the visit in a log entry: what went well, what did not go well, what advice they would offer for future visits, and what concerns they have about future visits. This creates a log entry in the care recipient's case file, as shown in Figure 3, which helps primary family caregivers understand the visit in more detail and can help other respite caregivers understand what to expect from an upcoming visit.

Schedule Management. Our design allows both caregivers to cross-reference schedules and desired availabilities easily and automatically sync schedules. Checking to see if an appointment appears on a respite caregiver's schedule (See Figure 4) can help mitigate concerns primary family caregivers expressed about schedule mix-ups. This design would be useful in managing care when multiple respite caregivers are involved.

\section{Accessing Technology}

Many respite care agencies use a primarily paper-based information sharing system because some care recipients, most notably those who lived in rural areas, had weak internet and cell reception. Additionally, since our participants used a number of different devices from desktops to smartphones, a single-platform design would not meet their needs.

Our design addresses these concerns by allowing certain critical functions such as drafting a log entry or viewing saved contacts' profiles to occur without an internet connection and plans to create interfaces for computers, tablets, and smartphones that are compatible with one another. Critical information about a client is available to respite caregivers offline to prevent issues respite caregivers who already used ICTs through their agency reported. The screen designs included in the margins are specifically for iPhone, but the features are designed to be generalized to other platforms easily.

\section{CONCLUSION}

This work focuses on understanding how trust and communication issues between primary family and respite caregivers could be mitigated by sharing information through ICTs. Previous healthcare, $\mathrm{HCl}$ and $\mathrm{CSCW}$ research has focused on the relatively long-term relationships between patients, physicians, and/or primary family caregivers, but our work has extended this to respite care. We uncovered the primary needs stakeholders identified through interviews, although our sample is too small to make broad generalizations. Our work also offers significant design implications for future ICTs. While our design focuses on communication between those caregivers, it includes a function to find proper respite caregivers, as existing apps provide. Our design can be improved through UX testing and efforts to reach a broader group of participants with an even more diverse set 
"I've heard a lot of negative comments from [Company]. Especially when it comes to long-term. You know, people not showing up, people being late, people being messy, the house not being left clean. All sorts of little issues.”

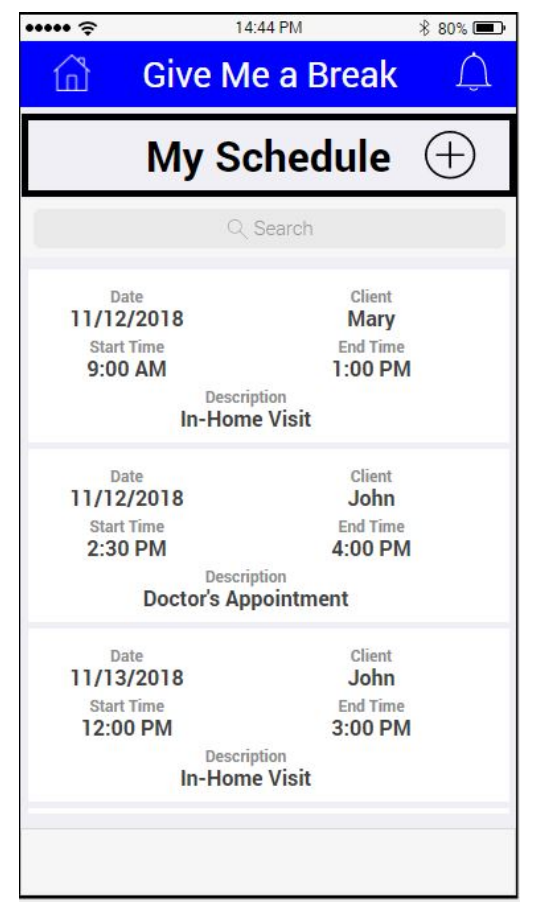

Figure 4: A respite care caregiver's schedule. Appointments are listed in order. Schedules and availabilities are accessible through a user's profile. of backgrounds and needs. Participants also expressed other needs or wishes for ICTs that were not in the scope of this project, which we hope to address through future work.

\section{ACKNOWLEDGMENTS}

This material is based upon work supported by the National Science Foundation under Grant No.CNS 1560276. Any opinions, findings, and conclusions or recommendations expressed in this material are those of the author(s) and do not necessarily reflect the views of the National Science Foundation. We thank Dr. Patrick C. Shih and Dr. Kay Connelly for their guidance throughout this project, and the Proactive Health Informatics Research Experience for Undergraduates at Indiana University Bloomington. We acknowledge and appreciate the time the participants in this study took out of their busy schedules. We thank the reviewers for CHI 2019 for their time and feedback.

\section{REFERENCES}

[1] Yunan Chen, Victor Ngo, and Sun Young Park. 2013. Caring for caregivers. In Proceedings of the 2013 conference on Computer supported cooperative work - CSCW '13. 91. https://doi.org/10.1145/2441776.2441789

[2] Hannah Chimowitz, Macda Gerard, Alan Fossa, Fabienne Bourgeois, and Sigall K. Bell. 2018. Empowering Informal Caregivers with Health Information: OpenNotes as a Safety Strategy. Joint Commission Journal on Quality and Patient Safety 44, 3 (2018), 130-136. https://doi.org/10.1016/j.jcjq.2017.09.004

[3] Pin Sym Foong, Shengdong Zhao, Felicia Tan, and Joseph Jay Williams. 2018. Harvesting caregiving knowledge: Design considerations for integrating volunteer input in dementia care. In Proceedings of the $2018 \mathrm{CHI}$ Conference on Human Factors in Computing Systems - CHI '18. 79. https://doi.org/10.1145/3173574.3173653

[4] Jamie Harrison, Rob Innes, and T. D. Zwanenberg. 2003. Rebuilding Trust in Healthcare. Radcliffe Publishing. 198 pages.

[5] Institute of Medicine. 2008. Retooling for an Aging America: Building the Health Care Workforce. National Academies Press (US), Washington, DC. 316 pages. https://doi.org/10.17226/12089

[6] Yun-Hee Jeon, Henry Brodaty, and Jon Chesterson. 2005. Respite care for caregivers and people with severe mental illness: literature review. Journal of Advanced Nursing 49, 3 (2005), 297-306. https://doi.org/10.1111/j.1365-2648.2004.03287.x

[7] Jiro Kawakita. 1991. The original KJ method. Kawakita Research Institute, Tokyo.

[8] Linda C. McSwiggan, Judith Marston, Martin Campbell, Timothy B. Kelly, and Thilo Kroll. 2017. Information-sharing with respite care services for older adults: a qualitative exploration of carers' experiences. Health \& Social Care in the Community 25, 4 (2017), 1404-1415. https://doi.org/10.1111/hsc. 12440

[9] National Alliance for Caregiving and AARP. 2015. Caregiving in the U.S. https://www.caregiving.org/caregiving2015/

[10] Tom Ongwere, Gabrielle Cantor, Sergio Ramirez Martin, Patrick C Shih, James Clawson, and Kay Connelly. 2018. Design Hotspots for Care of Discordant Chronic Comorbidities: Patients' Perspectives. In Proceedings of the Nordic Conference on Human-Computer Interaction. ACM, 571-583.

[11] Anselm Strauss and Juliet M. Corbin. 1990. Basics of qualitative research: Grounded theory procedures and techniques. Sage Publications, Inc., Newbury Park, CA, US.

12] Naomi Yamashita, Hideaki Kuzuoka, Keiji Hirata, and Takashi Kudo. 2013. Understanding the conflicting demands of family caregivers caring for depressed family members. In Proceedings of the SIGCHI Conference on Human Factors in Computing Systems - CHI '13. 2637-2646. https://doi.org/10.1145/2470654.2481365 